\title{
Institutional Delivery Service Utilization and Associated Factors Among Women in West Central Ethiopia
}

\author{
Meseret Ifa*, Elias Teferi \\ Department of Public Health, College of Medicine and Health Sciences, Ambo University, Ambo, Ethiopia \\ Email address: \\ mesol.ifa@gmail.com (M. Ifa), eliasteferi2015@gmail.com (E. Teferi) \\ ${ }^{*}$ Corresponding author
}

\section{To cite this article:}

Meseret Ifa, Elias Teferi. Institutional Delivery Service Utilization and Associated Factors Among Women in West Central Ethiopia. Science Journal of Public Health. Vol. 7, No. 2, 2019, pp. 38-43. doi: 10.11648/j.sjph.20190702.12

Received: February 1, 2019; Accepted: March 18, 2019; Published: April 12, 2019

\begin{abstract}
Pregnancy and child birth related complications are main causes of death and disability among women of childbearing age in developing countries. The Sub-Saharan Africa accounts for more than half of the global maternal deaths and most of these deaths occur during labor and following child birth from preventable causes that can be averted by professional intervention. Hence, the aim of this study was to assess the level of institutional delivery service utilization and associated factors among women who gave birth in West Central Ethiopia. A community based cross-sectional study was conducted from January 10 to February 10, 2014. Quantitative data were collected using a structured questionnaire and analyzed using SPSS V.20.0. Descriptive statistics were used to present the data and logistic regression was applied to check the association between the dependent and independent variables. A total of 410 women were included in the study of whom $70.7 \%$ had given birth their last baby at health facilities. Women with secondary and above educational status (AOR $[95 \% \mathrm{CI}]=4.525[95 \% \mathrm{CI}: 1.831-11.180]$ ), those who had an access to Radio and TV (AOR [95\%CI] $=3.214[1.361-7.591]$ ), women from families with average monthly income greater than 1,000.00 Ethiopian Birr (AOR [95\%CI] $=3.300[1.555-$ 6.999]), and women who had antenatal care follow up during their last pregnancy (AOR [95\%CI] $=2.409$ [1.088-5.335]) were more likely to utilize institutional delivery service. In conclusion, a significant number of women did not give birth their last baby at health facilities. Maternal educational status, access to mass media, family income status and antenatal care visit were important predictors of institutional delivery service utilization. Increasing Health Extension Workers' involvement in improving community awareness, and women empowerment through education and economic development are important measures to improve institutional delivery service utilization.
\end{abstract}

Keywords: Institutional Delivery, Ambo Town, Delivery Service

\section{Introduction}

Globally, about 289,000 women died from pregnancy related complications in 2013, and $99 \%$ of the deaths occurred in developing countries. The sub-Saharan Africa countries alone constituted for about $62 \%$ of these deaths; and Ethiopia is one of the countries with high number of maternal deaths (676 maternal deaths per 100,000 live births in 2011) [1, 2].

Most of the maternal deaths are attributed to hemorrhage, high blood pressure, infection, abortion related complications and obstructed labor that occur during child birth and within 24 hours following delivery $[1,3,4]$. Improved delivery care is one of the important strategies of preventing most of the maternal deaths [5-7] as it increases the chance to prevent, detect and manage health problems before they get worsened $[2,8]$.

Maternal mortality tends to be higher where skilled attendance at birth is relatively low. In 2011 the proportion of births attended by skilled birth attendants in rural and urban settings of developing countries were reported to be $53 \%$ and $84 \%$ respectively. With its low level of skilled attendance at birth, Sub Saharan Africa has the world's highest maternal mortality ratio [5].

Studies conducted in different parts of Ethiopia show varying levels of institutional delivery service utilization that ranges from $4.9 \%$ in Boricha district of Sidama zone [9] to $80 \%$ in Debre Markos town [10]. The Ethiopian 
Demographic and Health Survey of 2011 report shows that only $10 \%$ ( $4 \%$ in rural and $50 \%$ in urban) of deliveries took place at health facilities whereas the majority of mothers gave birth at home [2]. These findings show that there is low institutional delivery service utilization in the country with significant difference between the urban and rural settings. Differences in the characteristics of the study participants and factors related to the study settings and health service delivery system could be major reasons for the variation. This may signify the need to consider local situations that affect institutional delivery service utilization to design intervention activities that can improve the uptake of the service. However, there was limited information on the level of institutional delivery service utilization and its associated factors at the current study area.

Thus, this study was intended to assess the level of institutional delivery service utilization and associated factors in Ambo town to provide essential information for health managers and decision makers in the process of maternal health improvement.

\section{Methods}

\subsection{Study Design and Setting}

A community based cross-sectional study was conducted on randomly selected women who gave birth in the last two years prior to the study period from January 10 to February 10, 2014 in Ambo town, West Central Ethiopia. Ambo town is located 114 kilometers away from Addis Ababa to the west and had three local administrative structures called Kebeles. The town had one public hospital and two public health centers that provide institutional delivery service during the data collection period.

\subsection{Sample Size and Sampling Technique}

The sample size was calculated using a single population proportion formula considering the proportion of institutional delivery service utilization $(\mathrm{P})$ to be $50 \%, 95 \%$ Confidence Interval, 5\% margin of error (d) and non-response rate of $10 \%$ that made the final sample size 422 .

A systematic random sampling was applied to select the study participants after census was conducted to identify women who gave birth in the last two years and sampling frame was prepared.

\subsection{Data Collection Method and Quality Control}

The data were collected by using structured questionnaire that was adapted after reviewing different literatures. The questionnaire was prepared in English and translated to Afan Oromo language which was then pre-tested on similar population in neighboring town to check for its clarity and consistency, and necessary modifications were made on the questionnaire before data were collected. Two days training was given for ten data collectors and five supervisors in order to create common understanding on the procedure and approaches to be used during data collection; addressing issues such as describing the objectives of the study, confidentiality of information, participants' right, informed consent and the general techniques of data collection.

\subsection{Data Management and Analysis}

The collected data were entered in to Epi Data 3.1 and exported to SPSS version 20.0 for analysis. The data were summarized by calculating percentage, mean and standard deviation to describe the socio-demographic characteristics of the study participants and other important variables. To identify the association between the dependent and independent variables, initially binary logistic regression was done. Then variables that became statistically significant with the dependent variable were moved to multiple logistic regressions after which independent predictors were declared at p-value less than 0.05 .

\subsection{Ethical Considerations}

Ethical clearance was obtained from Ambo University, College of Medicine and Health Sciences Ethical Review Committee. Support letters were written to Ambo town municipality and health office. The study participants were informed about the objectives of the study and informed consent was obtained from each study participant before data were collected. The confidentiality was kept strictly during data collection, handling, storage and analysis.

\section{Results}

\subsection{Socio-demographic Characteristics of the Study Participants}

A total of 410 women who gave birth in the last two years participated in the study that makes the response rate $97.2 \%$. The mean age of the respondents was $27.3+5.3$ years. Majority of the respondents belong to the Oromo Ethnic group (83.2\%). Married and Orthodox religion followers account for $84 \%$ and $49.3 \%$ of the respondents respectively (Table 1).

Table 1. Socio-demographic characteristics of the study participants, Ambo town, West Shoa Zone, Oromia Region, Ethiopia, 2014.

\begin{tabular}{lll}
\hline Variables & No. & Percent \\
\hline Age (in Years) & & \\
$<20$ & 25 & 6.1 \\
$20-24$ & 100 & 24.4 \\
$25-29$ & 166 & 40.5 \\
$30-34$ & 72 & 17.6 \\
$35-39$ & 36 & 8.8 \\
$40-44$ & 9 & 2.2 \\
$45-49$ & 2 & 0.5 \\
Religion & & \\
Orthodox & 202 & 49.3 \\
Protestant & 188 & 45.9 \\
Muslim & 16 & 3.9 \\
Others & 4 & 1 \\
Ethnicity & & \\
Oromo & 341 & 83.2 \\
Amhara & 60 & 14.6 \\
\hline
\end{tabular}




\begin{tabular}{lll}
\hline Variables & No. & Percent \\
\hline Tigre & 5 & 1.2 \\
Others & 4 & 1 \\
Marital status & & \\
Married & 345 & 84.1 \\
Divorced & 30 & 7.3 \\
Widowed & 23 & 5.6 \\
Never married & 12 & 2.9 \\
Mother's educational status & & \\
No formal education & 60 & 14.6 \\
Primary education & 160 & 39.0 \\
Secondary education and above & 190 & 46.3 \\
Occupational status of the respondent & & \\
Employed & 161 & 39.3 \\
Merchant & 75 & 18.3 \\
Housewife & 159 & 38.8 \\
Others & 15 & 3.7 \\
Husband's educational status/Formal & & \\
education/(n=345) & & \\
Yes & 299 & 86.7 \\
No & 46 & 13.3 \\
Occupational status of the husband & & \\
Employed & 179 & 51.9 \\
Merchant & 129 & 37.4 \\
Householder & 16 & 4.6 \\
Others & 21 & 6.1 \\
Access to mass media in a week (Radio and TV) & & \\
Had no access at all & 70 & 17.1 \\
Had access to either radio or TV & 193 & 47.1 \\
Had access to both radio and TV & 147 & 35.9 \\
Monthly income (in Ethiopian birr)* & & \\
Less than 400 & 97 & 23.7 \\
400 to 1000 & 141 & 34.4 \\
Greater than 1000 & 172 & 42.0 \\
Total family size & & \\
Four to three five & 146 & 35.6 \\
More than five & 196 & 47.8 \\
\hline & 68 & 16.6 \\
\hline The ave ex & & \\
\hline
\end{tabular}

* The average exchange rate for 1dollar during the data collection period was about 19.3003 Ethiopian Birr

\subsection{Obstetric History of the Study Participants}

Sixty three $(15.4 \%)$ of the respondents reported that they had delivered their first child at the age of 19 or younger. About two-third $(66.6 \%)$ of the respondents reported that they had two or more children at the time of the study. About one in five $(23.7 \%)$ of the women reported that their last pregnancy was not planned. About $15.1 \%$ of the respondents did not attend Antenatal Care (ANC) during their last pregnancy and $54.3 \%$ of those who attended ANC had four or more visits.

\subsection{Institutional Delivery Service Utilization}

Two hundred ninety $(70.7 \%)$ of the study participants reported that they gave birth their last baby at health facility assisted by health care providers while the remaining reported that they gave birth at home. The 120 respondents who gave birth at home mentioned they were assisted by different individuals during delivery: mother/mother-in-law 72 (60\%), traditional birth attendants $19(15.8 \%)$, neighbors $(22.5 \%)$ and health extension workers $(2 \%)$. Among mothers who reported home delivery, $27.5 \%$ of them stated that they believe delivery is an easy process while $18 \%$ of them reported that they had no awareness on the importance of delivery service at health facility (Figure 1).

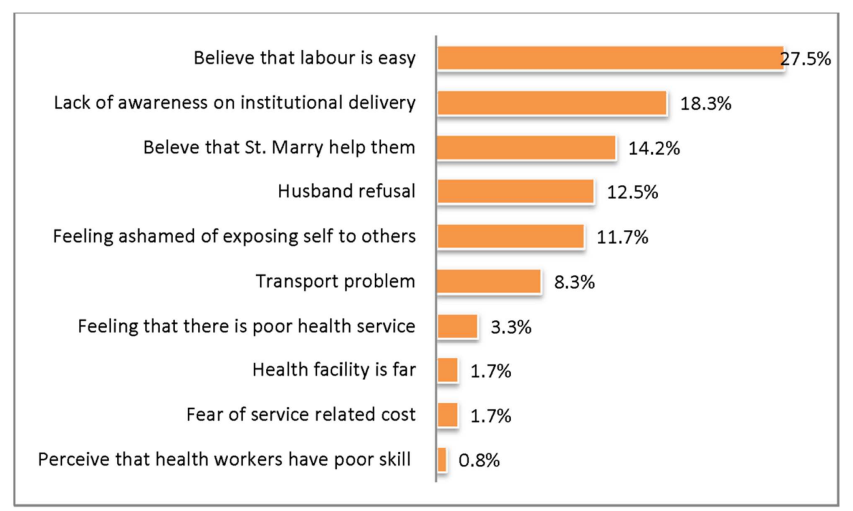

Figure 1. The percentage distribution of reasons provided for home delivery by women who gave birth their last baby at home in Ambo town, West Shoa Zone, Oromia Region, Ethiopia, 2014.

\subsection{Factors Associated with Institutional Delivery Service Utilization}

The binary logistic regression analysis shows that the woman's and husband's educational status, access to mass media, family income status and ANC visit during the last pregnancy have significant association with institutional delivery service utilization. Then multiple logistic regression analysis was conducted to identify the independent predictors of institutional delivery service utilization. The analysis showed that the level of maternal education, access to mass media, family income status and ANC visit were the independent predictors of institutional delivery service utilization.

The study shows that women who attended primary education (AOR $[95 \% \mathrm{CI}]=3.970 \quad[1.667-9.456])$ and secondary education and more $(\mathrm{AOR}[95 \% \mathrm{CI}]=4.525[95 \%$ CI: 1.831-11.180]) were more likely to utilize institutional delivery service compared to those with no formal education. Women from families with average monthly income greater than 1,000.00 Ethiopian Birr were found to be 3.3 times more likely to have delivered their last baby in health facilities compared to those who were from family with monthly income of less than 400 Ethiopian Birr (AOR [95\% CI] = 3.300 [1.555-6.999]).

Women who reported having either functional radio or TV as source of information in their home were about 2.7 times (AOR $[95 \% \mathrm{CI}]=2.681[1.830-6.075])$ more likely to give birth at health facility than their counter parts and those who had both Radio and TV were about 3 times (AOR [95\%CI] $=3.214$ [1.361-7.591]) more likely to utilize institutional delivery service compared to those who had neither radio nor TV.

Women who had at least one ANC visit during their last pregnancy $(\mathrm{AOR}[95 \% \mathrm{CI}]=2.409[1.088-5.335])$ were about 2.4 times more likely to use institutional delivery service when compared with those who did not attend ANC (Table 2). 
Table 2. Factors associated with institutional delivery service utilization in Ambo town, West Shoa zone, Oromia Region, Ethiopia, 2014.

\begin{tabular}{|c|c|c|c|c|}
\hline \multirow{2}{*}{ Variables } & \multicolumn{2}{|c|}{ Institutional delivery } & \multirow{2}{*}{ COR $(95 \% C I)$} & \multirow{2}{*}{ AOR (95\% C.I) } \\
\hline & Yes & No & & \\
\hline \multicolumn{5}{|l|}{ Mother's educational status } \\
\hline No formal education & 32 & 28 & 1 & 1 \\
\hline Primary education & 121 & 39 & $5.949(3.128,11.316)$ & $3.970(1.667,9.456)^{*}$ \\
\hline Secondary education and above & 137 & 53 & $5.407(2.905,10.064)$ & $4.525(1.831,11.180)^{*}$ \\
\hline \multicolumn{5}{|c|}{ Husband education /formal education/ } \\
\hline Yes & 234 & 63 & $2.857(1.498,5.451)$ & $1.094(0.474,2.524)$ \\
\hline No & 26 & 20 & 1 & 1 \\
\hline \multicolumn{5}{|c|}{ Family income per month (in Ethiopian Birr) } \\
\hline Less than 400 & 46 & 51 & 1 & 1 \\
\hline 400 to 1000 & 109 & 32 & $4.845(2.742,8.561)$ & $2.760(1.330,5.725)^{*}$ \\
\hline Greater than 1000 & 135 & 37 & $5.126(2.967,8.857)$ & $3.300(1.555,6.999)^{*}$ \\
\hline \multicolumn{5}{|l|}{ Access to media per week } \\
\hline No exposure at all & 22 & 48 & 1 & 1 \\
\hline Access to radio or $\mathrm{TV}$ & 149 & 44 & $4.531(2.540,8.084)$ & $2.681(1.83,6.075)^{*}$ \\
\hline Access to radio and TV & 119 & 28 & $5.850(3.134,10.920)$ & $3.214(1.361,7.591)^{*}$ \\
\hline \multicolumn{5}{|c|}{ ANC attendance for recent pregnancy } \\
\hline Yes & 260 & 88 & $4.352(2.483,7.626)$ & $2.409(1.088,5.335)^{*}$ \\
\hline No & 30 & 32 & 1 & 1 \\
\hline
\end{tabular}

$*$ P-value $<0.05$

\section{Discussion}

Among the 410 study participants, 290 (70.7\%) reported that they gave birth their last baby in health facilities. Though this finding is in line with finding from study conducted in a rural district of Tanzania (74.5\%) [11], it is lower than the prevalence of institutional delivery service utilization for urban settings in developing countries in 2011which was $84.4 \%$ [5] and findings from studies conducted in other towns of Ethiopia; Bahir Dar city (78.8\%) [12] and Debre Markos town $(80.14 \%)$ [10]. However, the proportion of institutional delivery service utilization in the study area is higher than findings from study conducted in Pakistan [13], and studies conducted in Boricha (9) and Goba (14) districts in Ethiopia. The differences in the level of institutional delivery service utilization could be explained by the possible variation in the characteristics of the study populations and service related factors in the respective study areas.

The study has shown that the educational status of the women is a determinant factor of institutional delivery service utilization. Mothers who had attended primary school and secondary school were about 3.97 and 4.5 times more likely to use institutional delivery service respectively when compared to those who had no formal education. This is similar to findings from other studies conducted in Ethiopia $[9,10,12,14,15]$ and Pakistan [13]. This could be explained by the fact that education increases access to information including pregnancy related risks and its management, and also improves negotiation skill to seek care when need arises. Likewise, mothers who had access to media (Radio and/or TV) were more likely to use institutional delivery service when compared to those who had neither. This is similar to findings from other studies [16, 17]. One possible explanation could be that mass media such as Radio and TV can increase the awareness of the mother and her family members on the need of maternal health care that may improve their attitude and decision making skill.

Woman's family income status was the other factor identified to have significant effect on the decision to choose health facility for child birth. The probability to give birth in the health facility was high with increased family income. Women from families with monthly income of greater than 1,000.00 Ethiopian Birr were about 3.3 times more likely to utilize institutional delivery service compared to those who were from family with average monthly income of 400 Ethiopian birr or less. This is supported by a study conducted in another town in Ethiopia [16]. Though delivery care in the country during the study period was free of charge, this could be explained by the fact that economic status could have various impacts on the process of decision making and accessing health care services. There could be indirect costs such as transportation that some women may not afford to reach the service area. The other possible explanation is the possible association between wealth and access to information. Increase in economic status could create opportunities to obtain better information from media and educational centers.

The study shows that the majority of institutional delivery service users had history of ANC visit during their last pregnancy. Women who had at least one ANC during their last pregnancy were 2.4 times more likely to have delivered their last baby in health facilities. This is in line with findings from other studies conducted in Ethiopia [10, 16, 17] and other countries $[11,13,18-20]$. This could be due to increase in awareness on pregnancy and delivery related risks and importance of health care through health education that is provided during ANC visits. In addition, ANC attendance could improve their familiarity with health facility environment and health care providers that my build their confidence to seek health care during delivery. 


\section{Limitation of the Study}

The fact that cross-sectional study design cannot identify temporal relationship between dependent and independent variables, its inability to establish the causal effect relationship and the possibility of recall bias need to be considered in interpreting the findings of the study.

\section{Conclusion and Recommendations}

A considerable number of women had given birth at home unattended by skilled personnel. Woman's educational status, access to mass media, family income status and ANC visit were found to be independent predictors of institutional delivery service utilization. Hence, there is a need of continuous awareness creation on the importance of institutional delivery service utilization at the community level using different approaches and Health Extension Workers can play a major role in this process. Promoting ANC service utilization and women empowerment through improved access to education and economic development are also important actions needed to improve the utilization of institutional delivery service in the area.

\section{Authors' Contributions}

Both authors wrote the proposal, participated in data collection, analyzed the data, drafted, read and approved the final manuscript.

\section{Competing Interests}

The authors declare that they have no competing interests.

\section{Acknowledgements}

The authors are thankful to Ambo University for providing them ethical approval and financial support for the study. They would also like to address their gratitude to all the study participants.

\section{References}

[1] World Health Organization, UNICEF. Trends in maternal mortality: 1990 to 2013: estimates by WHO, UNICEF, UNFPA, The World Bank and the United Nations Population Division: executive summary. World Health Organization; 2014.

[2] ICF C. Central Statistical Agency [Ethiopia] and ICF International. Ethiopia Demographic and Health Survey. 2011.

[3] Khan KS, Wojdyla D, Say L, Gülmezoglu AM, Van Look PF. WHO analysis of causes of maternal death: a systematic review. The lancet. 2006 Apr 1; 367 (9516):1066-74.

[4] Hogan MC, Foreman KJ, Naghavi M, Ahn SY, Wang M, Makela SM, Lopez AD, Lozano R, Murray CJ. Maternal mortality for 181 countries, 1980-2008: a systematic analysis of progress towards Millennium Development Goal 5. The lancet. 2010 May 8; 375(9726):1609-1623.

[5] United Nations. The Millennium Development Goals Report. New York. 2013.

[6] Campbell OM, Graham WJ, Lancet Maternal Survival Series steering group. Strategies for reducing maternal mortality: getting on with what works. The lancet. 2006; 368(9543):1284-99.

[7] Goldie SJ, Sweet S, Carvalho N, Natchu UC, Hu D. Alternative strategies to reduce maternal mortality in India: a cost-effectiveness analysis. PLoS medicine. 2010; 7(4): e1000264.

[8] Hatt L, Stanton C, Ronsmans C, Makowiecka K, Adisasmita A. Did professional attendance at home births improve early neonatal survival in Indonesia?. Health policy and planning. 2009; 24(4):270-278.

[9] Gudura TT, Debiso AT, Gudura TT. Factors associated with Institutional delivery in Boricha district of Sidama zone, southern Ethiopia. International Journal of Public Health Science (IJPHS). 2014; 3(4):224-230.

[10] Bayu H, Adefris M, Amano A, Abuhay M. Pregnant women's preference and factors associated with institutional delivery service utilization in Debra Markos Town, North West Ethiopia: a community based follow up study: BMC Pregnancy and Childbirth (2015) 15:15. DOI 10.1186/s12884015-0437-z.

[11] Exavery A, KantéAM, Njozi M, Tani K, Doctor HV, Hingora A, Phillips JF. Access to institutional delivery care and reasons for home delivery in three districts of Tanzania. International journal for equity in health. 2014; 13(1), 48.

[12] Abeje G, Azage M, Setegn T. Factors associated with Institutional delivery service utilization among mothers in Bahir Dar City administration, Amhara region: a community based cross sectional study. Reproductive Health. 2014; 11(1):22.

[13] Agha and Carton: Determinants of institutional delivery in rural Jhang, Pakistan. International Journal for Equity in Health. 2011. 10:31.

[14] Daniel BogaleOdo, DesalegnMarkosShifti. Institutional Delivery Service Utilization and Associated Factors among Child Bearing Age Women in GobaWoreda, Ethiopia. Journal of Gynecology and Obstetrics. Vol. 2, No. 4, 2014, pp. 63-70. doi: 10.11648/j.jgo.20140204.14.

[15] Teferra et al.: Institutional delivery service utilization and associated factors among mothers who gave birth in the last 12 months in Sekela District, North West of Ethiopia: A community - based cross sectional study. BMC Pregnancy and Childbirth. 2012; 12:74.

[16] Birmeta K, Dibaba Y, Woldeyohannes D. Determinants of maternal health care utilization in Holeta town, central Ethiopia. BMC health services research. 2013; 13(1):256.

[17] Mengesha ZB, Biks GA, Ayele TA, Tessema GA, Koye DN. Determinants of skilled attendance for delivery in Northwest Ethiopia: a community based nested case control study. BMC Public Health. 2013; 13(1):130.

[18] Trujillo JC, Carrillo B, Iglesias WJ. Relationship between professional antenatal care and facility delivery: an assessment of Colombia. Health policy and planning. 2013; 29 (4):443-449. 
[19] Babalola SO. Factors associated with use of maternal health services in Haiti: a multilevel analysis. Revista Panamericana de Salud Pública. 2014; 36:1-09.
[20] Sharma SR, Poudyal AK, Devkota BM, Singh S. Factors associated with place of delivery in rural Nepal. BMC Public Health. 2014; 14(1):306. 